\title{
Forcing of Infinity and Algebras of Distributions of Binary Semi-isolating Formulas for Strongly Minimal Theories
}

\author{
Sergey V. Sudoplatov \\ Sobolev Institute of Mathematics, Academician Koptyug avenue, 4, 630090, Novosibirsk, Russia \\ Novosibirsk State Technical University, K.Marx avenue, 20, 630073, Novosibirsk, Russia \\ Novosibirsk State University, Pirogova street, 2, 630090, Novosibirsk, Russia \\ *Corresponding Author: sudoplat@math.nsc.ru
}

Copyright (C)2014 Horizon Research Publishing All rights reserved.

\begin{abstract}
We apply a general approach for distributions of binary isolating and semi-isolating formulas to the class of strongly minimal theories. For this aim we introduce and use the notion of forcing of infinity. Structures associated with binary formulas, in strongly minimal theories, and containing compositions and Boolean combinations are characterized: a list of basic structural properties for these structures, including the forcing of infinity, is presented, and it is shown that structures satisfying this list of properties are realized in strongly minimal theories.
\end{abstract}

Keywords Structure of Binary Semi-isolating Formulas, Forcing of Infinity, Strongly Minimal Theory

\section{Mathematics Subject Classification (2000) 03C07, 03G15, 20N02, 08A02, 08A55}

\section{Introduction}

Algebras and structures associated with isolating and semi-isolating formulas of a theory are introduced in $[1,2]$. These derived structures allow to find basic structural properties, to describe theories and their models on binary level, as well as to classify them. We apply the general approach for distributions of formulas to an important class of elementary theories: the class of strongly minimal theories [3] for which all definable sets are either finite or cofinite. Recall that strongly minimal theories on binary level were investigated in $[4,5,6]$.

The paper is organized as follows. In Section 2 we recall basic definitions for structures of distributions of formulas. In Section 3 we introduce the notion of forcing of infinity, recall the notion of strongly minimal theory, and illustrate the forcing of infinity on some basic examples including strongly minimal theories. In Section
4, using the forcing of infinity, we characterize the class of structures for distributions of formulas: (1) a list of basic structural properties for these structures is presented (Theorem 4.1); (2) applying a syntactic generic construction it is shown that structures satisfying this list of properties are realized in strongly minimal theories (Theorem 4.2).

\section{Algebras of distributions of binary isolating and semi- isolating formulas}

We denote first-order theories by $T$, structures by $\mathcal{M}$, and the universe of $\mathcal{M}$ by $M$.

Definition $[1,2,7,8]$. Let $T$ be a complete theory, $\mathcal{M}$ be a model of $T: \mathcal{M} \models T$. Consider types $p(x), q(y) \in$ $S(\emptyset)$ (i. e., complete consistent sets of formulas of $T$ with at most one free variable, $x$ and $y$ respectively, and without parameters) realized in $\mathcal{M}$, and all $(p, q)$ preserving, or semi-isolating, formulas $\varphi(x, y)$ of $T$, i. e., formulas for which there is $a \in M$ such that $\models p(a)$ and $\varphi(a, y) \vdash q(y)$. Now, for each such a formula $\varphi(x, y)$, we define a binary relation $R_{p, \varphi, q}=\{(a, b) \mid \mathcal{M} \models p(a) \wedge$ $\varphi(a, b)\}$. If $(a, b) \in R_{p, \varphi, q},(a, b)$ is called a $(p, \varphi, q)$-arc. If $\varphi(a, y)$ is principal (over $a)$, the $(p, \varphi, q)$-arc $(a, b)$ is also principal.

If $\varphi(x, y)$ is a $(p \leftrightarrow q)$-formula, i. e., it is both a $(p \rightarrow q)$ - and a $(q \rightarrow p)$-formula then the set $[a, b]=\{(a, b),(b, a)\}$ is said to be a $(p, \varphi, q)$-edge. If the $(p, \varphi, q)$-edge $[a, b]$ consists of principal $(p, \varphi, q)$ - and $(q, \varphi(y, x), p)$-arcs then $[a, b]$ is a principal $(p, \varphi, q)$-edge.

$(p, \varphi, q)$-arcs and $(p, \varphi, q)$-edges are called arcs and edges respectively if we say about fixed or some formula $\varphi(x, y)$. If $(a, b)$ is a $(p, \varphi, q)$-arc such that the pair $(b, a)$ is not an arc for any $(q, p)$-formula, then $(a, b)$ is called irreversible. 
For types $p(x), q(y) \in S(\emptyset)$, we denote by $\operatorname{PF}(p, q)$ the set

$\{\varphi(x, y) \mid \varphi(a, y)$ is a principal formula, $\varphi(a, y) \vdash q(y)$,

$$
\text { where } \models p(a)\} \text {. }
$$

Let $\operatorname{PE}(p, q)$ be the set of pairs of formulas $(\varphi(x, y), \psi(x, y)) \in \operatorname{PF}(p, q)$ such that for any (some) realization $a$ of $p$ the sets of solutions for $\varphi(a, y)$ and $\psi(a, y)$ coincide. Clearly, $\operatorname{PE}(p, q)$ is an equivalence relation on the set $\operatorname{PF}(p, q)$. Notice that each $\operatorname{PE}(p, q)$ class $E$ corresponds to either a principal edge or to an irreversible principal arc connecting realizations of $p$ and $q$ by some (any) formula in $E$. Thus the quotient $\mathrm{PF}(p, q) / \mathrm{PE}(p, q)$ is represented as a disjoint union of sets $\operatorname{PFS}(p, q)$ and $\operatorname{PFN}(p, q)$, where $\operatorname{PFS}(p, q)$ consists of $\operatorname{PE}(p, q)$-classes corresponding to principal edges and $\operatorname{PFN}(p, q)$ consists of $\operatorname{PE}(p, q)$-classes corresponding to irreversible principal arcs.

Let $T$ be a complete theory without finite models, $U=$ $U^{-} \dot{\cup}\{0\} \dot{\cup} U^{+}$be an alphabet of cardinality $\geq|S(T)|$ and consisting of negative elements $u^{-} \in U^{-}$, positive elements $u^{+} \in U^{+}$, and zero 0 . As usual, we write $u<0$ for any $u \in U^{-}$and $u>0$ for any $u \in U^{+}$. The set $U^{-} \cup\{0\}$ is denoted by $U \leq 0$ and $U^{+} \cup\{0\}$ is denoted by $U^{\geq 0}$. Elements of $U$ are called labels.

Let $\nu(p, q): \operatorname{PF}(p, q) / \operatorname{PE}(p, q) \rightarrow U$ be injective $l a$ belling functions, $p(x), q(y) \in S(\emptyset)$, for which negative elements correspond to the classes in

$$
\operatorname{PFN}(p, q) / \operatorname{PE}(p, q)
$$

and non-negative elements correspond to the classes in $\operatorname{PFS}(p, q) / \mathrm{PE}(p, q)$ such that 0 is defined only for $p=$ $q$ and is represented by the formula $(x \approx y), \nu(p)=$ $\nu(p, p)$. We additionally suppose that $\rho_{\nu(p)} \cap \rho_{\nu(q)}=\{0\}$ for $p \neq q$ (where, as usual, we denote by $\rho_{f}$ the image of the function $f$ ) and $\rho_{\nu(p, q)} \cap \rho_{\nu\left(p^{\prime}, q^{\prime}\right)}=\emptyset$ if $p \neq q$ and $(p, q) \neq\left(p^{\prime}, q^{\prime}\right)$. Labelling functions with the properties above as well families of these functions are said to be regular. Below we shall consider only regular labelling functions and their regular families.

We denote by $\theta_{p, u, q}(x, y)$ formulas in $\operatorname{PF}(p, q)$ with a label $u \in \rho_{\nu(p, q)}$. If the type $p$ is fixed and $p=q$ then the formula $\theta_{p, u, q}(x, y)$ is denoted by $\theta_{u}(x, y)$.

For types $p_{1}, p_{2}, \ldots, p_{k+1} \in S^{1}(\emptyset)$ and sets $X_{1}, X_{2}, \ldots, X_{k} \subseteq U$ of labels we denote by

$$
P\left(p_{1}, X_{1}, p_{2}, X_{2}, \ldots, p_{k}, X_{k}, p_{k+1}\right)
$$

the set of all labels $u \in U$ corresponding to formulas $\theta_{p_{1}, u, p_{k+1}}(x, y)$ satisfying, for realizations $a$ of $p_{1}$ and some $u_{1} \in X_{1}, \ldots, u_{k} \in X_{k}$, the following condition:

$$
\theta_{p_{1}, u, p_{k+1}}(a, y) \vdash \theta_{p_{1}, u_{1}, p_{2}, u_{2}, \ldots, p_{k}, u_{k}, p_{k+1}}(a, y),
$$

where

$$
\begin{gathered}
\theta_{p_{1}, u_{1}, p_{2}, u_{2}, \ldots, p_{k}, u_{k}, p_{k+1}}(x, y) \rightleftharpoons \\
\rightleftharpoons \exists x_{2}, x_{3}, \ldots x_{k}\left(\theta_{p_{1}, u_{1}, p_{2}}\left(x, x_{2}\right) \wedge \theta_{p_{2}, u_{2}, p_{3}}\left(x_{2}, x_{3}\right) \wedge \ldots\right. \\
\left.\ldots \wedge \theta_{p_{k-1}, u_{k-1}, p_{k}}\left(x_{k-1}, x_{k}\right) \wedge \theta_{p_{k}, u_{k}, p_{k+1}}\left(x_{k}, y\right)\right) .
\end{gathered}
$$

Thus the Boolean $\mathcal{P}(U)$ of $U$ is the universe of an algebra of distributions of binary isolating formulas with $k$-ary operations $P\left(p_{1}, \cdot, p_{2}, \cdot, \ldots, p_{k}, \cdot, p_{k+1}\right)$, where $p_{1}, \ldots, p_{k+1} \in S^{1}(\emptyset)$. This algebra has a natural restriction to any family $R \subseteq S^{1}(\emptyset)$ forming $I_{\mathcal{R}}$-structure, where $\mathcal{R}$ is a set of labels for 1-types in $R$.

If all types $p_{i}$ equal to a type $p$ then we write $P_{p}\left(X_{1}, X_{2}, \ldots, X_{k}\right)$ and $P_{p}\left(u_{1}, u_{2}, \ldots, u_{k}\right)$ as well as $\left\lfloor X_{1}, X_{2}, \ldots, X_{k}\right\rfloor_{p}$ and $\left\lfloor u_{1}, u_{2}, \ldots, u_{k}\right\rfloor_{p}$ instead of $P\left(p_{1}, X_{1}, p_{2}, X_{2}, \ldots, p_{k}, X_{k}, p_{k+1}\right)$ and $P\left(p_{1}, u_{1}, p_{2}, u_{2}, \ldots, p_{k}, u_{k}, p_{k+1}\right) \quad$ respectively. We omit the index $\cdot{ }_{p}$ if the type $p$ is fixed and write simply $u_{1} \cdot u_{2}$ or $u_{1} u_{2}$ instead of $\left\lfloor u_{1}, u_{2}\right\rfloor$.

Extending the set $U$ of labels by positive and negative labels for semi-isolating formulas as well as by neutral labels $u^{\prime} \in U^{\prime}$ (collecting irreversible arcs and principal edges in a set of solutions of a semi-isolating formula), we define similar $\mathrm{SI}_{\mathcal{R}}$-structures $S I$ for semi-isolating formulas.

For types $p(x), q(y) \in S(\emptyset)$, we denote by $\operatorname{SICF}(p, q)$ the set of $(p \rightarrow q)$-formulas $\varphi(x, y)$ such that $\{\varphi(a, y)\}$ is consistent for $\models p(a)$. Let $\operatorname{SICE}(p, q)$ be the set of pairs of formulas $(\varphi(x, y), \psi(x, y)) \in \operatorname{SICF}(p, q)$ such that for any (some) realization $a$ of $p$ the sets of solutions for $\varphi(a, y)$ and $\psi(a, y)$ coincide. Clearly, $\operatorname{SICE}(p, q)$ is an equivalence relation on the $\operatorname{set} \operatorname{SICF}(p, q)$. Notice that each $\operatorname{SICE}(p, q)$-class $E$ corresponds to either a set of $(p, \varphi, q)$-edges, or a set of irreversible $(p, \varphi, q)$ arcs, or simultaneously a set of $(p, \varphi, q)$-edges and of irreversible $(p, \varphi, q)$-arcs linking realizations of $p$ and $q$ by any (some) formula $\varphi$ in $E$. Thus the quotient $\operatorname{SICF}(p, q) / \operatorname{SICE}(p, q)$ is represented as a disjoint union of sets $\operatorname{SICFE}(p, q), \quad \operatorname{SICFA}(p, q)$, and $\operatorname{SICFM}(p, q)$, where $\operatorname{SICFE}(p, q)$ consists of $\operatorname{SICE}(p, q)$-classes corresponding to sets of edges, $\operatorname{SICFA}(p, q)$ consists of $\operatorname{SICE}(p, q)$-classes corresponding to sets of irreversible arcs, and $\operatorname{SICFM}(p, q)$ consists of $\operatorname{SICE}(p, q)$-classes corresponding to sets containing edges and irreversible arcs.

The sets $\operatorname{SICF}(p, p), \quad \operatorname{SICE}(p, p), \quad \operatorname{SICFE}(p, p)$, $\operatorname{SICFA}(p, p)$, and $\operatorname{SICFM}(p, p)$ are denoted by $\operatorname{SICF}(p)$, $\operatorname{SICE}(p), \operatorname{SICFE}(p), \operatorname{SICFA}(p)$, and $\operatorname{SICFM}(p)$ respectively.

Let $T$ be a complete theory without finite models, $U=U^{-} \dot{\cup}\{0\} \dot{\cup} U^{+} \dot{\cup} U^{\prime}$ be an alphabet of cardinality $\geq|S(T)|$ and consisting of negative elements $u^{-} \in U^{-}$, positive elements $u^{+} \in U^{+}$, neutral elements $u^{\prime} \in U^{\prime}$, and zero 0 . As usual, we write $u<0$ for any $u \in U^{-}$ and $u>0$ for any $u \in U^{+}$. The set $U^{-} \cup\{0\}$ is denoted by $U \leq 0$ and $U^{+} \cup\{0\}$ is denoted by $U^{\geq 0}$. Elements of $U$ are called labels.

Let $\quad \nu(p, q): \operatorname{SICF}(p, q) / \operatorname{SICE}(p, q) \rightarrow U$ be injective labelling functions, $p(x), q(y) \in S(\emptyset)$, for which negative elements correspond to the classes in $\operatorname{SICFA}(p, q) / \operatorname{SICE}(p, q)$, positive elements and 0 correspond to the classes in $\operatorname{SICFE}(p, q) / \operatorname{SICE}(p, q)$ such that 0 is defined only for $p=q$ and is represented by the formula $(x \approx y)$, and neutral elements code the classes in $\operatorname{SICFM}(p, q) / \operatorname{SICE}(p, q), \nu(p) \rightleftharpoons \nu(p, p)$. We additionally assume that $\rho_{\nu(p)} \cap \rho_{\nu(q)}=\{0\}$ for $p \neq q$ where, as usual, we denote by $\rho_{f}$ the image of the function $f$ ) and $\rho_{\nu(p, q)} \cap \rho_{\nu\left(p^{\prime}, q^{\prime}\right)}=\emptyset$ if $p \neq q$ and $(p, q) \neq\left(p^{\prime}, q^{\prime}\right)$. Labelling functions with the properties above as well families of these functions are said to be regular. Below we shall consider only regular labelling functions and their regular families.

The labels, corresponding to isolating formulas, are 
said to be isolating whereas each label in $\bigcup_{p, q \in S^{1}(\emptyset)} \rho_{\nu(p, q)}$ is semi-isolating. By the definition, each isolating label belongs to $U^{-} \dot{\cup}\{0\} \dot{\cup} U^{+}$, i. e., it is not neutral.

We denote by $\theta_{p, u, q}(x, y)$ formulas in $\operatorname{SICF}(p, q)$ with a label $u \in \rho_{\nu(p, q)}$. If the type $p$ is fixed and $p=q$ then the formula $\theta_{p, u, q}(x, y)$ is denoted by $\theta_{u}(x, y)$.

Note that if $\theta_{p, u, q}(x, y)$ and $\theta_{q, v, p}(x, y)$ are formulas witnessing that for realizations $a$ and $b$ of $p$ and $q$ respectively the pairs $(a, b)$ and $(b, a)$ belong to $\mathrm{SI}_{\{p, q\}}$, then the formula $\varphi(x, y)=\theta_{p, u, q}(x, y) \wedge \theta_{q, v, p}(y, x)$ witnesses that $[a, b]$ is a $(p, \varphi, q)$-edge. If the edge $[a, b]$ is principal and $\theta_{p, u, q}(a, y)$ is an isolating formula such that $\models \theta_{p, u, q}(a, b), \models p(a)$, then the label $u$ is invertible and the label $v \in U^{\geq 0}$ corresponds uniquely to $u$ such that $\theta_{q, v, p}(b, y)$ is an isolating formula with $\models \theta_{q, v, p}(b, a)$, and vice versa. The labels $u$ and $v$ are reciprocally inverse and are denoted by $v^{-1}$ and $u^{-1}$ respectively. In general case, each label $u \in U^{\geq 0}$ has a (nonempty) set of inverse labels in $U^{\geq 0}$, denoted also by $u^{-1}$. Note that independently on a label $u \in U^{\geq 0}$, for which a formula $\theta_{p, u, q}(x, y)$ witnesses that $[a, b]$ is a $(p, \varphi, q)$-edge, the set $u^{-1}$ includes all labels $v \in U^{\geq 0}$ such that $[b, a]$ is a $\left(q, \theta_{q, v, p}, p\right)$-edge.

Neutral labels correspond, for instance, the formulas $\theta_{p, u, q}(x, y) \vee \theta_{p, v, q}(x, y)$, where $u<0$ and $v \geq 0$.

For types $p_{1}, p_{2}, \ldots, p_{k+1} \in S^{1}(\emptyset)$ and sets $X_{1}, X_{2}, \ldots, X_{k} \subseteq U$ of labels we denote by

$$
\operatorname{SI}\left(p_{1}, X_{1}, p_{2}, X_{2}, \ldots, p_{k}, X_{k}, p_{k+1}\right)
$$

the set of all labels $u \in \rho_{\nu\left(p_{1}, p_{k+1}\right)}$ corresponding to formulas $\theta_{p_{1}, u, p_{k+1}}(x, y)$ satisfying the condition (1) for realizations $a$ of $p_{1}$ and some $u_{1} \in X_{1} \cap \rho_{\nu\left(p_{1}, p_{2}\right)}, \ldots, u_{k} \in$ $X_{k} \cap \rho_{\nu\left(p_{k}, p_{k+1}\right)}$. In view of transitivity of semi-isolation, each formula $\theta_{p_{1}, u_{1}, p_{2}, u_{2}, \ldots, p_{k}, u_{k}, p_{k+1}}(x, y)$ has a label in $\rho_{\nu\left(p_{1}, p_{k+1}\right)}$.

Thus the Boolean $\mathcal{P}(U)$ of $U$ is the universe of an algebra $A$ of distributions of binary semi-isolating formulas with $k$-ary operations

$$
\mathrm{SI}\left(p_{1}, \cdot, p_{2}, \cdot, \ldots, p_{k}, \cdot, p_{k+1}\right),
$$

where $p_{1}, \ldots, p_{k+1} \in S^{1}(\emptyset)$. This algebra has a natural restriction to any family $R \subseteq S^{1}(\emptyset)$ as well as to the algebras of distributions of binary isolating formulas. Besides, if $U_{0}$ is a subalphabet of $U$ then the restriction of the universe of $A$ to the set $\mathcal{P}\left(U_{0}\right)$ and the restrictions for values of operations to the set $U_{0}$ forms, possibly partial, algebra $\left.A\right|_{U_{0}}$.

If all types $p_{i}$ equal to a type $p$ then we write $\mathrm{SI}_{p}\left(X_{1}, X_{2}, \ldots, X_{k}\right)$ and $\mathrm{SI}_{p}\left(u_{1}, u_{2}, \ldots, u_{k}\right)$ as well as $\left\lceil X_{1}, X_{2}, \ldots, X_{k}\right\rceil_{p}$ and $\left\lceil u_{1}, u_{2}, \ldots, u_{k}\right\rceil_{p}$ instead of

$$
\operatorname{SI}\left(p_{1}, X_{1}, p_{2}, X_{2}, \ldots, p_{k}, X_{k}, p_{k+1}\right)
$$

and

$$
\operatorname{SI}\left(p_{1}, u_{1}, p_{2}, u_{2}, \ldots, p_{k}, u_{k}, p_{k+1}\right)
$$

respectively. We omit the index $\cdot p$ if the type $p$ is fixed. In this case, we write $\theta_{u_{1}, u_{2}, \ldots, u_{k}}(x, y)$ instead of $\theta_{p, u_{1}, p, u_{2}, \ldots, p, u_{k}, p}(x, y)$.

Having the algebras $A$ we can consider the notions of si-ranks, Boolean operations for labels, relations of domination of labels with respect to $\vdash, \mathrm{SI}_{\mathcal{R}}$-structures, and POSTC $_{\mathcal{R}}$-structures collecting these attributes [2].

\section{Forcing of infinity and strongly minimal theories}

Let $T$ be a theory, $R \subseteq S^{1}(\emptyset)$ be a nonempty family, and $\nu(R)$ be a regular family of labelling functions for semi-isolating formulas forming a set $U$ of labels. Denote by $U_{\text {fin }}$ (respectively $U_{\text {cofin }}$ ) the set of labels $u$, each of which, being in $\bigcup_{p, q \in R} \rho_{\nu(p, q)}$, dominates a (co)finite set of labels in $\rho_{\nu(p, q)}$.

By the definition all almost deterministic labels (i.e., labels $u \in \rho_{\nu(p, q)}$ such that $\theta_{p, u, q}(a, y)$ has finitely many solutions for $\models p(a))$ belong to $U_{\text {fin }}$.

Definition. We say that a label $u \in \rho_{\nu(p, q)}$ forces an infinite set of solutions for the formula $\theta_{p, u, q}(a, y)$, $=p(a)$, if for any theory $T$ with a family $R$ of 1-types, containing $p$ and $q$ and having a POSTC $_{R}$-structure, including all labels that are dominated by $u$, the formula $\theta_{p, u, q}(a, y)$ has infinitely many solutions.

By the definition a label $u \in \rho_{\nu(p, q)}$ forces an infinite set of solutions if and only if for any $n \in \omega$ and some $n$ elements $a_{1}, \ldots, a_{n}$ in the set of solutions of $\theta_{p, u, q}(a, y)$ (in an arbitrary structure), where $\models p(a)$, there exists new element $a_{n+1}$, for which $\models \theta_{p, u, q}\left(a, a_{n+1}\right)$ and links between $a_{n+1}$ and $a_{1}, \ldots, a_{n}$ are defined by some labels.

Clearly, almost deterministic labels do not force infinite sets of solutions and any label $u$, dominating infinitely many labels $v_{i}$, forces an infinite set of solutions. Moreover, each label $u \wedge \neg v_{i_{1}} \wedge \ldots \wedge \neg v_{i_{n}}$ also forces an infinite set of solutions. Another example with a label, which forces an infinite set of solutions, is presented by theory $\operatorname{Th}(\langle\mathbf{Q},<\rangle)$, for which any non-zero label (defining the strict order property) corresponds to formulas having only infinitely many solutions. An infinite set of solutions can be forced by labels in $U_{\text {fin }}$ for formulas in stable theories. Such an example is produced by any label corresponding to a special element of an infinite group for an everywhere finitely defined polygonometry [9].

Definition [3]. A theory $T$ is called strongly minimal if for any formula $\varphi(x, \bar{a})$ of language obtained by adding parameters of $\bar{a}$ (in some model $\mathcal{M} \models T$ ) to the language of $T$, either $\varphi(x, \bar{a})$, or $\neg \varphi(x, \bar{a})$ has finitely many solutions.

An example of strongly minimal theory with the forcing of infinite set is represented by structure $\langle M ; s\rangle$ with successor function $s$ (having exactly one preimage for any element, and do not having cycles). Since $\operatorname{Th}(\langle M ; s\rangle)$ has unique 1-type, there is a label $u \in U_{\text {cofin }}$ for the semi-isolating formula $(x \approx x)$. This label dominates infinitely many labels $v$ corresponding to formulas $\left(y \approx s^{n}(x)\right), n \in \mathbf{Z}$, and thus, $u$ forces an infinite set of solutions for the formulas $\theta_{u}(a, y), a \in M$.

\section{Results}

Theorem 4.1. For any strongly minimal theory $T$, the family $R \rightleftharpoons S^{1}(\emptyset)$ of 1-types, and a regular family $\nu(R)$ of labelling functions for semi-isolating formulas, the following conditions hold: 
(a) the POSTC $\mathrm{R}_{\mathcal{R}}$-structure $\mathcal{M}_{\nu(R)}$ consists of labels belonging to $U_{\text {fin }} \cup U_{\text {cofin }}$;

(b) there is unique type $r_{0} \in R$ having infinitely many realizations; in particular, any set $\rho_{\nu(p, q)}$ is finite, where $p, q \in R$ and $q \neq r_{0}$, and all labels $u \in \rho_{\nu(p, q)}$ are almost deterministic and belong to $U_{\text {fin }}$;

(c) if $R$ is finite, i. e., all types in $R$ are principal, then all non-zero labels are positive and all labels $u$, including zero, have complements $\bar{u}$, and for any pair of labels $u, \bar{u} \in \rho_{\nu\left(p, r_{0}\right)}$, exactly one of them is almost deterministic and, in particular, belongs to $U_{\text {fin }}$, and the other label marks a formula $\theta_{p, ; r_{0}}(a, y)$ with infinitely many solutions, where $=p(a)$, and belongs to $U_{\text {cofin }}$;

(d) if $R$ is infinite, i. e., $r_{0}$ is unique non-principal 1 type, then all non-zero labels, linking realizations of $r_{0}$ or realizations of types in $R \backslash\left\{r_{0}\right\}$, are positive, and labels, linking realizations of $r_{0}$ with realizations of types in $R \backslash$ $\left\{r_{0}\right\}$, are negative; in this case, if a label $u$ belongs to $\rho_{\nu\left(p, r_{0}\right)}$ then $u$ is positive or zero, almost deterministic, does not have complements and $p=r_{0}$, moreover, $U_{\text {fin }}=$ $U_{\text {cofin }}$ if $\rho_{\nu\left(r_{0}\right)}$ is finite, and $U_{\text {cofin }}=\emptyset$ if $\rho_{\nu\left(r_{0}\right)}$ is infinite;

(e) only labels in $\rho_{\nu\left(p, r_{0}\right)}$ with the principal type $r_{0}$ can force infinitely many solutions.

Proof. By the definition of strongly minimal theory, each formula $\varphi(a, y)$, where $\models p(a)$, (in particular, witnessing the semi-isolation for a type $q(y))$ has a finite or a cofinite set $\varphi(a, \mathcal{M})$ of solutions. For the finite set $\varphi(a, \mathcal{M})$, the label $u \in \rho_{\nu(p, q)}$, marking the formula $\varphi(x, y)$ with $\varphi(a, y) \vdash q(y)$ and $\models p(a)$ can dominate only finitely many labels in $\rho_{\nu(p, q)}$. If $\varphi(a, \mathcal{M})$ is cofinite, then the label $u \in \rho_{\nu(p, q)}$ for the formula $\varphi(x, y)$ dominates all labels in $\rho_{\nu(p, q)}$ except for a finitely many $u_{1}, \ldots, u_{k}$, and $u$ has the complement $\bar{u}$, which is obtained from $u$ by disjunctive attachment of labels $u_{i}$. Thus the condition (a) holds: all labels belong to $U_{\text {fin }} \cup U_{\text {cofin }}$.

Since $T$ is strongly minimal we also have that there is unique type $r_{0} \in R$, principal or non-principal, with infinitely many realizations: if there are finitely many 1-types it is implied by the property that models are infinite and there are no two principal formulas with infinitely many realizations, and if there are infinitely many 1-types, then the non-principal type $r_{0}(x)$, having infinitely many realizations by Compactness, is isolated by the set of all formulas $\neg \varphi(x)$, where $\varphi(x)$ are principal formulas and none of these formulas can not have infinitely many solutions.

Since the type $r_{0}$ with infinitely many realizations is unique, then any set $\rho_{\nu(p, q)}$ is finite, where $p, q \in R$ and $q \neq r_{0}$. Here all labels $u \in \rho_{\nu(p, q)}$ are almost deterministic and belong to $U_{\text {fin }}$. Thus, we have the condition (b).

If $r_{0}$ is isolated then all 1-types are isolated and by Proposition 1.1 [2] all non-zero labels are positive. Since each isolating formula $\varphi(x)$ has a label, all labels, including zero, have complements. In this case for any pair of labels $u, \bar{u} \in \rho_{\nu\left(p, r_{0}\right)}$, exactly one of these labels is almost deterministic and, in particular, belongs to $U_{\text {fin }}$, and the other label marks a formula $\theta_{p, \cdot, r_{0}}(a, y)$ with infinitely many solutions, where $\models p(a)$, and belongs to $U_{\text {cofin }}$. Hence, the condition (c) holds.

If $r_{0}$ is non-isolated, then all non-zero labels, linking realizations of $r_{0}$ are positive, since having a non- positive non-zero label $u$, linking realizations of $r_{0}$ we have the non-symmetric relation $\mathrm{SI}_{r_{0}}$ and as $r_{0}$ is nonisolated there are infinitely many solutions for the formula $\theta_{u}(x, a)$, where $\models r_{0}(a)$. This contradicts the strong minimality of theory $T$. By Proposition 1.1 [2], non-zero labels linking realizations of types in $R \backslash\left\{r_{0}\right\}$, are positive, and labels, linking realizations of $r_{0}$ with realizations of types in $R \backslash\left\{r_{0}\right\}$, are negative. In this case, since for non-principal type there are only relative complements, if a label $u$ belongs to $\rho_{\nu\left(p, r_{0}\right)}$, then $u$ is positive or zero, almost deterministic and does not have a complement. Moreover, $p=r_{0}$ since realizations of principal types cannot semi-isolate realizations of non-principal type $r_{0}$. If the set $\rho_{\nu\left(r_{0}\right)}$ is finite, then any label in $U_{\text {fin }}$ belongs to $U_{\text {cofin }}$ and vice versa, i. e., $U_{\text {fin }}=U_{\text {cofin }}$, and if $\rho_{\nu\left(r_{0}\right)}$ is infinite, then all labels are almost deterministic and $U_{\text {cofin }}=\emptyset$. Thus, the condition (d) holds. The condition (e) is implied by previous items. Theorem is proved.

If $\mathcal{M}$ is a POSTC $\mathcal{R}_{\mathcal{R}}$-structure and there is a theory $T$ with a family $R=S^{1}(\emptyset)$ and a regular family $\nu(R)$ of labelling functions for semi-isolating formulas such that $\mathcal{M}_{\nu(R)}=\mathcal{M}$, then we say that $\mathcal{M}$ is represented by $T$ and also say that $T$ represents the POSTC $_{\mathcal{R}}$-structure $\mathcal{M}$. If all types of $R$ are realized in a model $\mathcal{N}$ of $T$, then we say that $\mathcal{M}$ is represented by $\mathcal{N}$.

Note that the syntactic representability of POSTC $\mathcal{R}^{-}$ structure $\mathcal{M}$ (by a theory) is equivalent to the semantic representability of $\mathcal{M}$ (by a model).

Notice also that there is a representation $T$ for the POSTC $_{\mathcal{R}}$-structure $\mathcal{M}$ such that a label $u$ is almost deterministic if and only if $u$ does not force an infinite set of solutions.

Theorem 4.2. Let $\mathcal{M}$ be a POSTC $_{\mathcal{R}}$-structure satisfying the following conditions:

(a) $\mathcal{M}$ consists of labels belonging to $U_{\text {fin }} \cup U_{\text {cofin }}$;

(b) there is an element $r_{0} \in \mathcal{R}$ such that any set $\rho_{\mu(p, q)}$ is finite, where $p, q \in \mathcal{R}$ and $q \neq r_{0}$, and all labels $u \in$ $\rho_{\mu(p, q)}$ are almost deterministic (in some representation $\mathcal{N}$ of $\mathcal{M})$ and belong to $U_{\text {fin }}$;

(c) if $\mathcal{R}$ is finite then all non-zero labels are positive and all labels $u$, including zero, have complements $\bar{u}$, and for any pair of labels $u, \bar{u} \in \rho_{\mu\left(p, r_{0}\right)}$, exactly one of them is almost deterministic and, in particular, belongs to $U_{\text {fin }}$, and the other label marks a formula $\theta_{p, \cdot, r_{0}}(a, y)$ (for $\mathcal{N})$ with infinitely many solutions, where $\models p(a)$, and belongs to $U_{\text {cofin; }}$;

(d) if $\mathcal{R}$ is infinite then all non-zero labels, linking $r_{0}$ or elements of $R \backslash\left\{r_{0}\right\}$, are positive, and labels, linking $r_{0}$ with elements in $R \backslash\left\{r_{0}\right\}$, are negative; in this case, if a label $u$ belongs to $\rho_{\mu\left(p, r_{0}\right)}$, then $u$ is positive or zero, almost deterministic, does not have complements and $p=r_{0}$, moreover, $U_{\text {fin }}=U_{\text {cofin }}$ if $\rho_{\mu\left(r_{0}\right)}$ is finite, and $U_{\text {cofin }}=\emptyset$ if $\rho_{\mu\left(r_{0}\right)}$ is infinite;

(e) only labels in $\rho_{\mu\left(p, r_{0}\right)}$ and with $|\mathcal{R}|<\omega$ can force infinity.

Then there is a strongly minimal theory $T$ representing the $\mathrm{POSTC}_{\mathcal{R}}$-structure $\mathcal{M}$ and having unique 1-type $r_{0}$ with infinitely many realizations.

Proof. Consider the construction for the proof of Theorems 9.1 [1] and 8.1 [2]. We identify $\mathcal{R}$ with the set of 1-types for the required theory $T$. Now we add to the 
types describing links between elements with respect to binary relations $Q_{u}, u \in U$, an information for the cardinality of sets of solutions for formulas $\theta_{p, u, q}(a, y)$, where $=p(a)$. The generic construction for the class $\mathbf{T}_{0}$ of types guarantees that the generic theory of the language $\left\{Q_{u} \mid u \in U\right\}$ is strongly minimal and represents the POSTC $_{\mathcal{R}}$-structure $\mathcal{M}$. Theorem is proved.

\section{Acknowledgements}

The research was supported by RFBR (grant 12-0100460-a).

\section{REFERENCES}

[1] I. V. Shulepov, S. V. Sudoplatov. Algebras of distributions for binary isolating formulas of a complete theory, arXiv:1205.3473v1 [math.LO], 2012.

[2] S. V. Sudoplatov. Algebras of distributions for binary semi-isolating formulas of a complete theory, arXiv:1210.4049v1 [math.LO], 2012.

[3] J. T. Baldwin, A. H. Lachlan. On strongly minimal sets, J. Symbolic Logic, 1971, Vol.36, No.1, 79-96.
[4] A. A. Ivanov. The problem of finite axiomatizability for strongly minimal theories of graphs, and groups with more than one end, Algebra and Logic, 1989, Vol.28, No.2, 160-173.

[5] A. A. Ivanov. The problem of finite axiomatizability for strongly minimal theories of graphs, Algebra and Logic, 1989, Vol.28, No.3, 280-297.

[6] B. Herwig, S. Lempp, M. Ziegler. Constructive models of uncountably categorical theories, Proc. Amer. Math. Soc., 1999, Vol.127, No.12, 3711-3719.

[7] S. V. Sudoplatov. The Lachlan problem, Edition of Novosibirsk State Technical University, Novosibirsk, 2009. [in Russian; English version is available in: http://www.math.nsc.ru/ sudoplatov/lachlan_eng_03_ 09_2008.pdf]

[8] B. S. Baizhanov, S. V. Sudoplatov, V. V. Verbovskiy. Conditions for non-symmetric relations of semiisolation, Siberian Electronic Mathematical Reports, 2012, Vol.9, 161-184.

[9] S. V. Sudoplatov. Group polygonometries, Edition of Novosibirsk State Technical University, Novosibirsk, 2011, 2013. [in Russian] 